\title{
Avaliação de práticas e saberes sobre fitoterapia e automedicação entre graduandos de enfermagem: Um estudo transversal
}

\author{
Evaluation of practices and knowledge about phytotherapy and self-medication among nursing \\ graduates: A cross-section study \\ Evaluación de prácticas y conocimientos sobre fitoterapia y automedicación en los egresados en \\ enfermería: Un estudio transversal
}

\section{Resumo}

Objetivo: realizar um levantamento sobre o conhecimento e uso de fitoterápicos e/ou plantas medicinais e a automedicação, entre discentes do curso de enfermagem, de uma Universidade Pública no município de Campina Grande - PB. Metodologia: Foi aplicado um questionário semiestruturado com perguntas relativas ao perfil socioeconômico e acadêmico, indagações sobre práticas e saberes em fitoterapia e automedicação. Os dados foram tabulados em planilha Excel 2010 e analisados pelo software R, avaliando a distribuição numérica e percentual, além do teste qui-quadrado, para verificar associação entre variáveis, obteve-se uma significância de $5 \%(p<0,05)$. Resultados: os entrevistados $(n=86)$ cursavam entre o $1^{\circ}$ e $8^{\circ}$ período, do gênero feminino $(77,0 \%)$, utilizam o SUS para atendimentos de saúde $(66,7 \%)$ e naturais de Campina Grande-PB $(60,4 \%)$. Havendo usado plantas medicinais ou derivados $(66,3 \%)$, não possuíam formação em fitoterapia $(79 \%)$, realizavam automedicação $(83,4 \%)$ principalmente com: analgésicos (87,2\%). Familiares e amigos foram a principal fonte de informação sobre plantas medicinais e(ou) medicamentos alopáticos, $38,4 \%$ e $31,4 \%$, respectivamente. A análise de associação entre variáveis $(p<0,05)$, mostrou que a renda familiar influencia no uso da assistência em saúde do SUS e que indivíduos que usam fitoterápicos têm maior tendência em indicar os mesmos. Conclusão: os entrevistados possuem um perfil em termos de automedicação, incluindo fitoterápicos, muito parecido com a população em geral, o que ressalta a importância de desenvolverem-se estratégias de abordagem da fitoterapia ao longo da formação de profissionais de nível superior em saúde.

Palavras-chave: Fitoterapia; Automedicação; Estudantes de enfermagem; Epidemiologia.

\begin{abstract}
Objective: to conduct a survey on the knowledge and use of herbal medicines and/or medicinal plants and selfmedication among nursing students at a Public University in the city of Campina Grande - PB. Methodology: A semistructured questionnaire was applied with questions related to the socioeconomic and academic profile, questions about practices and knowledge in herbal medicine and self-medication. The data were tabulated in an Excel 2010 spreadsheet and analyzed using the $\mathrm{R}$ software, evaluating the numerical and percentage distribution, in addition to the chi-square test to verify the association between variables. Results: respondents $(n=86)$ attended between the 1st and 8th period, females $(77.0 \%)$ use the SUS for health care $(66.7 \%)$ and are from Campina Grande-PB (60.4\%). Having used medicinal plants or derivatives $(66.3 \%)$, they had no training in herbal medicine $(79 \%)$, performed self-medication $(83.4 \%)$, mainly with: analgesics $(87.2 \%)$. Family and friends were the main source of information about medicinal plants and/or allopathic medicines, $38.4 \%$ and $31.4 \%$, respectively. The analysis of association between variables $(\mathrm{p}<0.05)$ showed that family income influences the use of SUS health care and that individuals who use herbal medicines have a greater tendency to indicate them. Conclusion: respondents have a profile in terms of self-medication, including herbal
\end{abstract}


medicines, very similar to the general population, which highlights the importance of developing strategies to approach herbal medicine throughout the training of higher education health professionals.

Keywords: Phytotherapy; Self-medication; Nursing students; Epidemiology.

\section{Resumen}

Objetivo: realizar una encuesta sobre el conocimiento y uso de las hierbas medicinales/plantas medicinales y la automedicación en estudiantes de enfermería de una Universidad Pública de la ciudad de Campina Grande - PB. Metodología: Se aplicó un cuestionario semiestructurado con preguntas relacionadas con el perfil socioeconómico/académico, preguntas sobre prácticas/conocimientos en fitoterapia y automedicación. Los datos se tabularon en Excel 2010 y se analizaron mediante el software R, evaluando la distribución numérica y porcentual, y la prueba de chi-cuadrado para verificar la asociación entre variables, se obtuvo una significancia del $5 \%(\mathrm{p}<0.05)$. Resultados: encuestados $(\mathrm{n}=86)$ entre el $1^{\circ}$ y $8^{\circ}$ período, mujeres $(77,0 \%)$, que utilizan el SUS $(66,7 \%)$ y de Campina Grande-PB $(60,4 \%)$. Habiendo utilizado plantas medicinales o derivados $(66,3 \%)$, no tenían formación en fitoterapia (79\%), se automedicaba $(83,4 \%)$, principalmente con analgésicos $(87,2 \%)$. La familia y los amigos fueron la principal fuente de información sobre plantas medicinales y medicamentos alopáticos, $38,4 \%$ y $31,4 \%$, respectivamente. El análisis de asociación entre variables ( $\mathrm{p}<0.05$ ) mostró que el ingreso familiar influye en el uso de la atención de salud del SUS y que los individuos que usan medicamentos a base de hierbas tienen una mayor tendencia a indicarlos. Conclusión: los entrevistados tienen un conocimiento común en materia de automedicación y fitoterapia, lo que destaca la importancia de desarrollar estrategias para abordar estos temas a lo largo de la formación de los profesionales de la educación superior en salud.

Palabras clave: Fitoterapia; Automedicación; Estudiantes de enfermería; Epidemiologia.

\section{Introdução}

O uso de plantas medicinais é uma forma de tratamento de origem muito antiga, relacionada aos primórdios da terapêutica em saúde e fundamentada no acúmulo de informações e vivências por sucessivas gerações. "Assim como todos os medicamentos, os fitoterápicos devem ser caracterizados pelo conhecimento da sua eficácia, pelos riscos de seu uso, pela reprodutibilidade de efeitos e a constância de sua qualidade" (Brasil, 2015, p. 219).

Com a finalidade de evitar o uso inadequado dessa prática medicinal no Brasil, o Ministério da Saúde buscou estimular a inserção da fitoterapia, entre outras práticas integrativas e complementares em saúde (PICS), no Sistema Único de Saúde (SUS). Tal prática apresenta alta relevância, pois cerca de $80 \%$ da população mundial a utiliza em seus cuidados básicos de saúde. Nesse sentido, "o uso de plantas medicinais deve estar baseado em conhecimentos científicos que comprovem sua eficácia e segurança, além de estar respaldado em políticas públicas adequadas” (Gonçalves, 2017, p. 192).

Por mais que esteja teorizada através dos documentos que regem o SUS, a fitoterapia ainda não é uma prática consolidada na assistência, pois, tal como expõe Santos (2014), sua utilização foi relegada a um segundo plano em detrimento dos avanços tecnológicos e o processo de industrialização no século XX, bem como, do avanço da medicina moderno e crescente utilização de medicamentos sintéticos.

Por ser um conhecimento que é transmitido oralmente de geração em geração, os relatos sobre os benefícios das plantas medicinais prevalecem, em relação aos malefícios, e esses, em muitos casos, não são claramente definidos (Duarte, 2020). O sistema de assistência em saúde não tem sido capaz de promover mudanças significativas nessa situação. Para Maia et al. (2016), o pouco conhecimento dos profissionais de saúde em relação às plantas medicinais e a fitoterapia são, em sua maioria, adquiridos a partir da família e da própria comunidade na qual eles trabalham. Isso demonstra que tais profissionais não foram preparados adequadamente em seus respectivos cursos de graduação, não tendo em sua grade curricular a disciplina de fitoterapia.

Outro aspecto, ligado não somente às plantas medicinais e aos fitoterápicos, mas aos medicamentos alopáticos, é a automedicação. Essa prática é comum tanto na população em geral quanto no meio de profissionais e estudantes da área de saúde, configurando um tema relevante entre especialistas no próprio ambiente acadêmico (Gama, Oliveira, Beazussi \& Gama, 2017; Zampieron, Sheng, Gontijo \& Mueller, 2020).

"O acesso da população aos medicamentos que necessita, inclusive às plantas medicinais e fitoterápicos, com segurança, eficácia e qualidade, deve ocorrer nos diferentes níveis de complexidade do SUS, com ênfase na atenção básica” (Brasil, 2016, 
p. 60). Nesse nível de atenção, o enfermeiro possui um vínculo com a comunidade de forma a conhecer as suas características sociais e culturais, esse conhecimento aliado a autonomia que o enfermeiro possui para prescrever, durante a consulta de enfermagem, medicamentos fitoterápicos ou não, pode resultar em favor da resolução dos problemas de saúde dos usuários dos serviços, garantindo uma atenção holística do indivíduo (COFEN, 1986).

Nesse contexto, compreender as percepções dos futuros profissionais enfermeiros sobre a importância, o conhecimento e utilização da Fitoterapia, bem como os limites e riscos da automedicação, faz-se necessário, a fim de avaliar os aspectos da formação profissional que influenciam a prática de uso de fitoterápicos e/ou plantas medicinais, além de medicamentos em geral, e as atitudes que o futuro enfermeiro terá em seu exercício profissional, em aspectos ligados à farmacoterapia. Portanto, o objetivo deste trabalho foi realizar um levantamento sobre o conhecimento e uso de fitoterápicos e/ou plantas medicinais e a automedicação, entre os estudantes de graduação em enfermagem, da Universidade Federal de Campina Grande, no município de Campina Grande-PB.

\section{Metodologia}

Este estudo parte de uma base quantitativa, que segundo Pereira A. S. et al (2018) é a realização de uma coleta de dados numéricos, que podem ser analisados por técnicas matemáticas para realizar a previsão de acontecimentos e fenômenos. Além de, realizar uma pesquisa exploratória e descritiva com a participação de graduandos em enfermagem da Universidade Federal de Campina Grande (UFCG), no Centro de Ciências Biológicas e da Saúde (CCBS) em Campina Grande-PB. Os dados foram obtidos durante o mês de maio de 2018, através da aplicação de um questionário sem identificação e a ser preenchido pelo entrevistado. Esse instrumento de coleta de dados era composto por variáveis relacionadas à fitoterapia e à automedicação, bem como ao perfil socioeconômico dos sujeitos de pesquisa. Visando verificar possíveis necessidades de ajustes ao instrumento de coleta de dados, foi realizado um teste piloto com alunos do primeiro período de enfermagem. Com isso, foram feitos ajustes para aperfeiçoar esse instrumento de coleta de dados.

Quanto à amostragem dos participantes, essa se deu por conveniência. Como critérios de inclusão, tivemos: estar regularmente matriculado no curso de enfermagem do CCBS-UFCG no momento da coleta de dados e concordar em participar voluntariamente da pesquisa mediante a assinatura do Termo de Consentimento Livre e Esclarecido (TCLE). A amostra final se constituiu por 86 (oitenta e seis) discentes, o que correspondeu a 63,2\% do total de alunos matriculados no semestre letivo de 2018.1

Os dados foram tabulados em planilha Excel 2010 e analisados pelo software R, avaliando a distribuição numérica e percentual e, ainda, pelo teste do qui-quadrado, para verificação de associação entre variáveis, com o nível de significância de $5 \%(\mathrm{p}<0,05)$.

Esta pesquisa foi aprovada pelo Comitê de Ética e Pesquisa da UFCG - Hospital Universitário Alcides Carneiro no dia 25 de abril de 2018, número do parecer: 2.618.949

\section{Resultados}

A amostra desta pesquisa foi composta por $86(63,2 \%$ do total) discentes que estavam regularmente matriculados entre o primeiro e o oitavo períodos do Curso de Enfermagem do Centro de Ciências Biológicas e da Saúde (CCBS) da Universidade Federal de Campina Grande (UFCG), no semestre letivo de 2018.1. Conforme descrito na Tabela 1, o perfil dos discentes de enfermagem corresponde, majoritariamente, a pessoas do gênero feminino com idade entre 18 e 22 anos (83,3\%), solteiros $(92,4 \%)$, com renda familiar entre 1 e 3 salários-mínimos (60,6\%). Além disso, foi observado que $66,7 \%$ dos participantes são usuários do SUS e 60,4\% naturais de Campina Grande-PB. 
Tabela 1. Distribuição (numérica e percentual) dos entrevistados quanto às variáveis socioeconômicas. Curso de Enfermagem, CCBS-UFCG, 2018.

\begin{tabular}{|c|c|c|c|c|}
\hline \multirow{3}{*}{ Variáveis } & \multicolumn{4}{|c|}{ Gênero } \\
\hline & \multicolumn{2}{|c|}{ Masculino } & \multicolumn{2}{|c|}{ Feminino } \\
\hline & $\mathbf{N}$ & $\%$ & $\mathbf{N}$ & $\%$ \\
\hline \multicolumn{5}{|l|}{$\begin{array}{r}\text { FAIXA ETÁRIA EM } \\
\text { ANOS COMPLETOS }(\mathrm{N}=86)\end{array}$} \\
\hline $18-22$ & 16 & $80,0 \%$ & 55 & $83,3 \%$ \\
\hline $23-29$ & 2 & $10,0 \%$ & 8 & $12,1 \%$ \\
\hline 30 ou mais & 2 & $10,0 \%$ & 3 & $4,6 \%$ \\
\hline Total & 20 & $100,0 \%$ & 66 & $100,0 \%$ \\
\hline \multicolumn{5}{|l|}{$\begin{array}{l}\text { ESTADO CIVIL } \\
(\mathrm{N}=86)\end{array}$} \\
\hline Solteiro $(a)$ & 19 & $95,0 \%$ & 61 & $92,4 \%$ \\
\hline Casado (a) & 0 & $0,0 \%$ & 2 & $3,0 \%$ \\
\hline Divorciado $(a)$ & 1 & $5,0 \%$ & 0 & $0 \%$ \\
\hline Outro & 0 & $0,0 \%$ & 3 & $4,6 \%$ \\
\hline Total & 20 & $100,0 \%$ & 66 & $100,0 \%$ \\
\hline \multicolumn{5}{|l|}{$\begin{array}{l}\text { RENDA FAMILIAR } \\
(\mathbf{N}=86)\end{array}$} \\
\hline $\begin{array}{r}\text { Menor que } 1 \\
\text { salário-mínimo }(S M)\end{array}$ & 4 & $20,0 \%$ & 13 & $19,7 \%$ \\
\hline $1-3 S M$ & 13 & $65,0 \%$ & 40 & $60,6 \%$ \\
\hline Maior que 3 SM & 3 & $15,0 \%$ & 13 & $19,7 \%$ \\
\hline Total & 20 & $100,0 \%$ & 66 & $100 \%$ \\
\hline \multicolumn{5}{|l|}{$\begin{array}{c}\text { QUAL SERVIÇO } \\
\text { DE SAÚDE UTILIZA }(\mathbf{N}=86) \\
\end{array}$} \\
\hline$S U S$ & 17 & $85,0 \%$ & 44 & $66,7 \%$ \\
\hline $\begin{array}{l}\text { Plano de saúde } \\
\text { (privado) }\end{array}$ & 2 & $10,0 \%$ & 12 & $18,2 \%$ \\
\hline Ambos & 1 & $5,0 \%$ & 10 & $15,1 \%$ \\
\hline Total & 20 & $100,0 \%$ & 66 & $100,0 \%$ \\
\hline
\end{tabular}

Fonte: Dados da pesquisa.

A associação entre renda familiar e tipo de serviço de saúde utilizado, foi uma das correlações com significância estatística, na nossa pesquisa. Percebe-se claramente (Tabela 2) que a grande maioria dos discentes com renda familiar de até 3 salários-mínimos, utilizam exclusivamente o Sistema Único de Saúde (SUS), quando estão enfermos.

Tabela 2. Associação estatisticamente significativa* entre as variáveis "renda familiar" e "serviço de saúde utilizado", conforme relato dos entrevistados.

\begin{tabular}{|c|c|c|c|c|}
\hline \multirow{2}{*}{ Renda Familiar } & \multicolumn{3}{|c|}{ Serviço de Saúde } & \multirow{2}{*}{ Total } \\
\hline & SUS & Privado & Ambos & \\
\hline$<$ Salário-mínimo & 15 & 0 & 2 & 17 \\
\hline 1-3 Salários-mínimos & 39 & 7 & 7 & 53 \\
\hline > 3 Salários-mínimos & 6 & 7 & 3 & 16 \\
\hline Total & 60 & 14 & 12 & 86 \\
\hline
\end{tabular}

* Considerando um nível de significância de 0,05 (5\%). Valor-p = 0,006788.

Fonte: Dados da pesquisa. 


\subsection{Práticas e saberes em Fitoterapia}

A análise sobre o conhecimento e práticas dos discentes foi direcionada inicialmente pela seguinte pergunta: Um conhecimento prévio sobre a Fitoterapia e Plantas Medicinais está associado com a relevância dada ao tema entre os discentes? Assim, constatou-se que apenas $21,0 \%$ dos discentes haviam tido alguma formação em fitoterapia durante a graduação. Sendo $11,6 \%$ com uma disciplina optativa de $30 \mathrm{~h}$, outros $9,4 \%$ que tinham tido algum conhecimento baseado em minicursos, palestras e eventos. Do total de participantes, $23,3 \%$ consideram o tema importante para a formação do profissional de saúde, 4,7\% o consideram irrelevante e $72,1 \%$ não responderam por não possuir formação/conhecimento sobre o assunto.

Entre os graduandos de Enfermagem entrevistados, a maioria (66,3\%) relatou utilizar (ou já ter utilizado) fitoterápicos ou plantas medicinais para tratar alguma enfermidade. Em se tratando da frequência de uso dos fitoterápicos e plantas medicinais, os discentes se distribuíram nas seguintes categorias de respostas, com seus respectivos percentuais: "uso esporádico" (34,9\%); "não uso" (33,7\%); uso raramente (25\%); "uso sempre que tenho necessidade" (34\%) e "uso todos os dias" (5,8\%). Além disso, entre os entrevistados, 50\% afirmam indicar ou já ter indicado o uso de plantas medicinais e/ou fitoterápicos a outras pessoas. Outra associação estatisticamente significativa $(\mathrm{p}<0,05)$ conforme nossos dados de pesquisa, foi o fato de que os jovens que relatam uso de plantas medicinais e/ou fitoterápicos têm maior tendência de indicar esses produtos para terceiros. Veja na (Tabela $3)$.

Tabela 3. Associação estatisticamente significativa* entre as variáveis "usar ou já ter usado plantas medicinais ou fitoterápicos (PM e F)" e "indicar ou já ter indicado plantas medicinais e derivados", conforme relato dos entrevistados.

\begin{tabular}{|c|c|c|c|}
\hline \multirow[t]{2}{*}{ Indica PM e F } & \multicolumn{2}{|c|}{ Usa PM e F } & \multirow[b]{2}{*}{ Total } \\
\hline & Não & Sim & \\
\hline Não & 24 & 19 & 43 \\
\hline Sim & 9 & 34 & 43 \\
\hline Total & 33 & 53 & 86 \\
\hline
\end{tabular}

* Considerando um nível de significância de 0,05 (5\%). Valor-p = 0,00088045 Fonte: Dados da pesquisa.

Além disso, uma parcela de 17,4\% dos estudantes não respondeu a esta pergunta de maneira satisfatória, mesmo sendo bem específica. Relatando apenas formas de preparo (chás, sucos, xarope) ou a finalidade para a qual utilizou (como, por exemplo: tratar gripe/dor abdominal e efeito calmante) ou ainda, citando apenas os tipos de plantas utilizadas (boldo, camomila, erva-doce). Ou seja, as variáveis foram utilizadas de forma dissociada. Conforme descrito na Tabela 4. 
Tabela 4. Distribuição dos entrevistados quanto relato da planta medicinal e respectiva finalidade de uso. Curso de Enfermagem, CCBS-UFCG, 2018.

\begin{tabular}{|c|c|c|c|}
\hline Planta citada/ \% & Nome científico & Finalidade de uso & Uso validado \\
\hline Alho $2,33 \%$ & Allium sativum & $\begin{array}{l}\text { Irritação na } \\
\text { garganta, gripe }\end{array}$ & $\begin{array}{l}\text { Coadjuvante no tratamento da hiperlipidemia e hipertensão } \\
\text { arterial, prevenção da aterosclerose }\end{array}$ \\
\hline Amora $1,16 \%$ & Morus nigra & Enjoo & Ação hipoglicemiante, anti-inflamatória e antimicrobiana \\
\hline Babosa $2,33 \%$ & Aloe sp. & $\begin{array}{l}\text { Tratar impinge e } \\
\text { furúnculo }\end{array}$ & $\begin{array}{l}\text { Antimicrobiana, antioxidante, anti-inflamatória, hidratante, } \\
\text { imunomoduladora, cicatrizante, etc }\end{array}$ \\
\hline Boldo $27,91 \%$ & Peumus boldus Molina & $\begin{array}{l}\text { Desconforto } \\
\text { abdominal, }\end{array}$ & Tratamento de dispepsias, como colorético \\
\hline $\begin{array}{l}\text { Camomila } \\
8,14 \%\end{array}$ & Matricaria chamomilla & $\begin{array}{l}\text { Tratar ansiedade, e } \\
\text { diarreia }\end{array}$ & $\begin{array}{l}\text { Tratamento de distúrbios gastrointestinais, inflamações da boca } \\
\text { e garganta, irritações na pele e mucosas }\end{array}$ \\
\hline Capim Santo $1,16 \%$ & Cymbopogon citratus & Calmante & Ação calmante e antiespasmódica suave \\
\hline Chuchu $1,16 \%$ & Sechium edule & Baixar pressão & Ação anti-hipertensiva \\
\hline Coentro $1,16 \%$ & Coriandrum sativum & Dores estomacais & Digestivo, antiespasmódico, antioxidante e antimicrobiano \\
\hline $\begin{array}{l}\text { Colônia } \\
2,33 \%\end{array}$ & Alpinia speciosa & Gripe e febre & Ação anti-hipertensiva e ação ansiolítica e sedativa. \\
\hline Copaíba $1,16 \%$ & Copaifera langsdorffii & Irritação na garganta & Cicatrizante e antimicrobiana. \\
\hline Erva-cidreira $5,81 \%$ & Melissa officinalis & Dores estomacais & $\begin{array}{l}\text { Atividade calmante e antiespasmódica suave, e atividade } \\
\text { analgésica }\end{array}$ \\
\hline Erva-doce $1,16 \%$ & Pimpinella anisum & Febre e cefaleia & $\begin{array}{l}\text { Antisséptico, antiespasmódico, diurético, laxante, estomacal e } \\
\text { tônica }\end{array}$ \\
\hline Eucalipto1,16\% & Eucalyptus & Febre & Analgésico, antisséptico, cicatrizante, expectorante etc. \\
\hline $\begin{array}{l}\text { Folha da Goiabeira } \\
5,81 \%\end{array}$ & Psidium guajava & Tratar diarreia & $\begin{array}{l}\text { Antidiarreica, antiespasmódica, antimicrobiana, } \\
\text { hipoglicemiante, antioxidante e antiagregante plaquetária }\end{array}$ \\
\hline $\begin{array}{l}\text { Folha da pimenta } \\
1,16 \%\end{array}$ & Capsicum spp. & Tratar furúnculo & $\begin{array}{l}\text { Combate parasitoses e infecções de pele (prurido, furúnculos } \\
\text { etc). }\end{array}$ \\
\hline $\begin{array}{l}\text { Folha de abacate } \\
1,16 \%\end{array}$ & Persea americana & Problemas renais & $\begin{array}{l}\text { Diurético, antirreumático, antidiarreico, anti-inflamatório, } \\
\text { antimicrobiano }\end{array}$ \\
\hline Hiperica $1,16 \%$ & Hypericum perforatum & Calmante & $\begin{array}{l}\text { Indicado para o tratamento de estados depressivos leves a } \\
\text { moderados }\end{array}$ \\
\hline $\begin{array}{l}\text { Lambedor de } \\
\text { Hortelã } 2,33 \%\end{array}$ & Mentha & Tosse & $\begin{array}{l}\text { Antiespasmódico, digestivo, antiemético, obstipante, } \\
\text { vermífugo, expectorante, antiinflamatório }\end{array}$ \\
\hline Limão $1,16 \%$ & Citrus limon & $\begin{array}{l}\text { Irritação no } \\
\text { estômago }\end{array}$ & $\begin{array}{l}\text { Bactericida, antiséptico, } \\
\text { antirreumático, calmante, diurético }\end{array}$ \\
\hline Erva-mate $1,16 \%$ & Ilex paraguariensis & Febrez e cefaleia & Diurético \\
\hline $\begin{array}{l}\text { Óleo de girassol } \\
1,16 \%\end{array}$ & Helianthus annuus L. & $\begin{array}{l}\text { Cicatrização de } \\
\text { feridas }\end{array}$ & Cicatrização de feridas \\
\hline Paniflora $1,16 \%$ & Passifloa incarnata L. & Calmante & Ação sedativa. \\
\hline $\begin{array}{l}\text { Pele da tomate } \\
1,16 \%\end{array}$ & Solanum lycopersicum & Tratar furúnculo & Não validado \\
\hline Pitanga $2,33 \%$ & Eugenia uniflora & Dores estomacais & $\begin{array}{l}\text { Ações antimicrobiana, antiparasitária, antioxidante, } \\
\text { hipotensora, diurética, anti-inflamatória e antidiarreica }\end{array}$ \\
\hline $\begin{array}{l}\text { Quebra Pedra } \\
2,33 \%\end{array}$ & Phyllanthus niruri & Pedra nos rins & $\begin{array}{l}\text { Tratamento de cálculo renal e urinário, diabetes, antiinfecciosa } \\
\text { e diurética }\end{array}$ \\
\hline $4,65 \%{ }^{\text {Romã }}$ & Punica granatum & Epiglotite & Antioxidantes, antimicrobiana e hipoglicêmica \\
\hline Umburana $1,16 \%$ & $\begin{array}{l}\text { Commiphora } \\
\text { leptophloeos }\end{array}$ & $\begin{array}{l}\text { Problemas } \\
\text { intestinais }\end{array}$ & Não validado \\
\hline $\begin{array}{l}\text { Xarope de Guaco } \\
2,33 \%\end{array}$ & Mikania glomerata & Tosse & Expectorante e antitussígeno. \\
\hline Mel 2,33\% & $\ldots$ & Gripe & Antitussígeno e tratamento de inflamações na garganta. \\
\hline
\end{tabular}

Fonte: Dados da pesquisa.

Quando questionados sobre o nível de satisfação a respeito das abordagens existentes na graduação sobre o tema, foi possível chegar às seguintes conclusões: apenas 29,1\% dos entrevistados estavam satisfeitos. Os demais estavam pouco 
satisfeitos $(45,3 \%)$ ou insatisfeitos $(14,0 \%)$ sobre como a fitoterapia foi abordada em sua graduação. Além disso, 11,6\% apresentaram-se indiferentes quanto ao tema.

\subsection{Automedicação com medicamentos industrializados}

O estudo também se propôs a avaliar a automedicação entre os discentes do curso de Enfermagem. Sendo assim verificou-se que dentre os discentes pesquisados $83,7 \%$ automedicavam-se. Ao se questionar os discentes sobre quais medicamentos utilizam sem prescrição médica e com quais finalidades, foi dada a opção de citar até 3 produtos. Assim, percebeuse que os principais são: os analgésicos com $87,2 \%$ dos relatos, antipiréticos (16,3\%) e anti-inflamatórios (11,6\%) conforme descrito na Tabela 5.

Tabela 5. Classificação dos medicamentos mais utilizados pelos discentes.

\begin{tabular}{c|r|r}
\hline $\begin{array}{c}\text { Grupo } \\
\text { farmacológico }\end{array}$ & \multicolumn{2}{|c}{ Frequência } \\
\hline Analgésico & 75 & $\%$ \\
\hline Antipirético & 14 & $87,2 \%$ \\
\hline Anti-inflamatório & 10 & $16,3 \%$ \\
\hline Antialérgico & 4 & $11,6 \%$ \\
\hline Antiemético & 4 & $4,7 \%$ \\
\hline Antibióticos & 4 & $4,7 \%$ \\
\hline Antigripal & 4 & $4,7 \%$ \\
\hline Probiótico & 3 & $4,7 \%$ \\
\hline Vitaminas & 2 & $3,5 \%$ \\
\hline Antidiarreico & 2 & $2,3 \%$ \\
\hline Antiácido & 1 & $2,3 \%$ \\
\hline Antihipertensivo & 1 & $1,2 \%$ \\
\hline
\end{tabular}

Fonte: Dados da pesquisa.

A frequência com que esses alunos se automedicam também foi quantificada: 68,6\% realizam automedicação esporadicamente, ou seja, apenas quando necessário. Esse valor é maior em relação ao uso esporádico de fitoterápicos $(34,9 \%)$ entre os participantes, conforme já apresentado. Ainda é importante mencionar que 6,9\% dos discentes, realizam automedicação diariamente; apesar de pequena essa proporção é preocupante, considerando os riscos provenientes dessa prática.

Da mesma forma que, quando do uso de fitoterápicos, os discentes responderam quais são as principais fontes de informação para a utilização sobre medicamentos alopáticos. A conclusão foi que 31,4\% dos estudantes utilizam mais de um meio de informação (associação entre internet, familiares e amigos, bula dos medicamentos). A segunda maior citação de fonte (23,3\%), foi a procura pela bula do produto. Em seguida, a busca de informação por meio familiares e amigos (17,4\%) e a busca apenas por meio da internet compõem 11,7\%. Por fim, a menor porcentagem, assim como no caso de fitoterápicos, refere-se a busca através de profissionais de saúde em uma proporção praticamente desprezível $(1,2 \%)$.

Sobre os riscos à saúde provenientes da automedicação, 80,2\% acreditam trazer malefícios à saúde, enquanto que, 19,8\% dos discentes alegaram não haver riscos.

\section{Discussão}

Conforme relatado, a amostra dessa pesquisa corresponde a $63,2 \%$ do total de alunos matriculados no momento da coleta. Enquanto no estudo de Silva (2014), 78 alunos, ou seja, 100\% dos matriculados participaram da pesquisa que teve por 
objetivo caracterizar a prática de automedicação e os fatores a ela associados entre estudantes ingressantes e concluintes do curso de graduação em enfermagem. Em um estudo semelhante avaliando o conhecimento dos acadêmicos de enfermagem relacionado à fitoterapia, Maia (2013) relata que dos 180 alunos que integravam o quadro estudantil da instituição, apenas 94 (52,2\%) aceitaram participar da pesquisa.

Quanto ao perfil socioeconômico dos participantes, podemos constatar que é semelhante ao encontrado na pesquisa de Correa (2018) acerca do conhecimento sobre fitoterapia e PICS, entre estudantes de enfermagem, que obteve idade média de 23,6 anos, estado civil solteiro em 82,7\% dos participantes, excetuando-se a renda familiar entre três e 10 salários-mínimos, enquanto esta pesquisa apresentou renda familiar majoritariamente até 3 salários-mínimos. Contrariando o discurso, de alguns segmentos da nossa sociedade, de que a Universidade Pública atenderia predominantemente jovens oriundos de camadas economicamente mais favorecidas da nossa população.

Acerca da utilização do SUS não foram encontrados outros trabalhos que também citaram essa variável, entretanto é interessante salientar que como a fitoterapia deve estar presente em todos os níveis de complexidade do SUS, o perfil do município frente à fitoterapia pode ser observado pelo conhecimento de seus usuários. Ademais, nos chama a atenção para o fato de que esse Sistema de Saúde, único no mundo, colabora também com o acesso e permanência de jovens no ensino superior público.

Também podemos observar que entre os indivíduos da pesquisa há uma predominância do gênero feminino (77\%). Esse fato também foi visto nos estudos de Maia (2013) e de Silva (2014) com 92,6\% e 87,1\% respectivamente. Isso, provavelmente, por influência de um estereótipo social, no qual as ações de enfermagem, por estarem diretamente relacionadas ao cuidado pelo outro, seriam tipicamente femininas, pois é papel das mães fornecer cuidados. Nos últimos anos essa ideia vem sendo desconstruída e um aumento gradativo dos homens nessa profissão vem sendo notado.

\subsection{Práticas e saberes em Fitoterapia}

A falta de capacitação sobre o tema, entre os sujeitos pesquisados, reflete-se no baixo percentual dos que reconhecem sua importância. Isso, obviamente, perpassa para a vida profissional do indivíduo, como é evidenciado no estudo de Oliveira (2017), em que 100\% dos enfermeiros das Unidades Básicas de Saúde (UBS) participantes, não possuíam conhecimento algum sobre a existência e aplicação da PNPIC e da PNPMF, e consequentemente, da possibilidade de prescrição de medicamentos fitoterápicos e promoção do uso racional de plantas medicinais.

Apesar do uso desses produtos não ser majoritariamente frequente entre os participantes dessa pesquisa, deve inspirar cuidado, pois é relativamente alto e se dá entre indivíduos, predominantemente, sem formação na área, ou seja, é possível que esse contato com a fitoterapia seja fundamentado apenas no conhecimento popular, e não científico, como é observado no estudo de Badke (2017).

Também foi observado que era mais comum que os indivíduos que faziam uso desses produtos também indicassem o uso para terceiros. Dessa forma, sugerindo uma continuação da prática de propagação de conhecimentos populares sem averiguação científica que, à semelhança da população em geral, é provavelmente movida pela crença cultural de que "o natural não faz mal". Assim, faz-se um alerta para a necessidade do uso de plantas medicinais e fitoterápicos de forma consciente e orientada por um profissional capacitado, como supracitado (Badke, 2017). Isso reforça a evidente necessidade de se trabalhar o tema da fitoterapia racional com esses graduandos, pois o comportamento de usar produtos terapêuticos derivados de plantas pode resultar em efeitos indesejáveis, não apenas aos próprios indivíduos, mas também para outras pessoas que façam parte dos seus relacionamentos sociais.

Dentre as plantas medicinais citadas, o boldo teve maior representatividade neste estudo, um resultado semelhante também foi encontrado numa pesquisa realizada com moradores do entorno do Parque Nacional Serra da Capivara - PI 
(Cansanção, 2017). O Peumus boldus Molina é o nome científico desta planta, e apesar de não compor a lista de fitoterápicos presentes na Relação Nacional de Medicamentos Essenciais (RENAME), é uma planta que está presente no Memento Fitoterápico da Farmacopeia brasileira, sendo indicada nas dispepsias funcionais e como estimulante hepático, além de possuir grande apreço na cultura popular para o tratamento de dores abdominais e distúrbios gastrointestinais (BRASIL, 2020). Associando a alta prevalência de relato de uso do Boldo com o fato de que as quatro plantas mais citadas também possuem efeitos terapêuticos no sistema digestório, afere-se que os problemas digestivos parecem ser o principal grupo de enfermidades entre os discentes entrevistados. Uma explicação poderia ser o fato de Campina Grande ser uma cidade universitária que acolhe muitos jovens provenientes de outras cidades, os quais, longe de suas famílias e morando em repúblicas estudantis, estariam provavelmente sujeitos a regimes alimentares de baixa qualidade (Brasil, 2017; Cansanção, 2017).

A camomila (Matricaria chamomilla), não está entre as mais citadas em estudos semelhantes, nem tampouco está na RENAME (BRASIL, 2020). Seu uso popular é associado a efeitos calmantes e sedativos, porém seu uso cientificamente validado refere-se ao tratamento de distúrbios gastrointestinais (distensão e constipação), inflamações da boca e garganta, irritações na pele e mucosa anal e genital e tratame nto de inflamação menor da pele (queimadura solar), feridas superficiais e furúnculos pequenos (BRASIL, 2015).

É importante enfatizar que apenas $29,1 \%$ dos entrevistados relataram estar satisfeitos com as abordagens da universidade para formação em fitoterapia. Um estudo com 157 profissionais de 66 equipes de UBS do município de Blumenau, identificou que o conhecimento destes, acerca da fitoterapia é limitado e informal, resultando em prescrições inadequadas ou inexistes de práticas fitoterápicas, como também da desvalorização por parte da equipe de saúde, que pode ser associado, à falta de formação adequada durante a graduação (Mattos, Camargo, Sousa \& Zeni, 2018). Outro resultado que reforça o impacto negativo da ausência da fitoterapia durante a formação de profissionais de saúde de nível superior, refere-se aos meios de informação que estes alunos têm a respeito do assunto, pois tais fontes influenciam de maneira direta em suas percepções e pensamentos sobre a importância da fitoterapia e seu uso racional. Nota-se que, a fonte de informação mais frequente $(38,4 \%)$, é justamente, a categoria dos "Familiares e Amigos", enquanto que os profissionais de saúde ocupam esse lugar apenas para $8,1 \%$ dos entrevistados. Tal fato nos mostra que, nesse aspecto, os entrevistados apenas reproduzem o padrão comportamental da população em geral. Segundo um estudo realizado por Badke (2017), o primeiro contato sobre este tema, geralmente, ocorre por meio da figura da mãe ao utilizar plantas medicinais na cura de doenças durante a infância dos filhos. Além disso, o estudo de Sampaio (2013), põe a figura da pessoa idosa como maior disseminadora de informações sobre o tema.

Esses dados reforçam a hipótese de que os conhecimentos que os entrevistados possuem não são cientificamente fundamentados. Acreditar que tudo aquilo que "é natural não faz mal" caracteriza-se como a mera reprodução de um pensamento popular que não deveria se aplicar a estudantes de ensino superior, principalmente, na área de saúde. Estes futuros profissionais vão trabalhar diretamente com os usuários dos serviços de saúde, principalmente na Atenção Primária, os quais majoritariamente se apropriam desse senso comum sobre a utilização de plantas medicinais, cabendo aos profissionais a intervenção para a promoção da fitoterapia racional (Barreto, 2015).

Assim, a falta de interesse sobre o tema, bem como a deficiência do sistema acadêmico, resulta em uma grande quantidade profissionais enfermeiros que não possuem conhecimento científico necessários para exercer seu importante papel de orientar e assistir a população, tampouco implementar a PNPMF na Atenção Primária da forma correta (Sampaio, 2013).

\subsection{Automedicação com medicamentos industrializados}

Entre os entrevistados desta pesquisa $83,7 \%$ relataram fazer automedicação principalmente com analgésicos, antipiréticos e anti-inflamatórios, dados semelhantes a esse foram encontrados no estudo de Zampieron (2019) em que a incidência de automedicação entre os acadêmicos da área de saúde entrevistados foi de 97,1\%. Do mesmo modo, os analgésicos 
se apresentavam em primeiro lugar com 87,6\% e os antiinflamatórios em segundo com 60,2\% (Zampieron, 2019), no estudo de Gama, Oliveira, Beazussi e Gama (2017) os analgésicos e antipiréticos em primeiro lugar (43,1\%), seguido de anticoncepcionais (10,5\%) e anti-inflamatórios (9,5\%), como também no de Lopes (2014) com 95,7\% e 85,3\%, respectivamente.

Apesar desses grupos farmacológicos serem isentos da obrigatoriedade de prescrição, conforme a legislação brasileira, faz-se necessário ressaltar os riscos decorrentes da sua utilização indiscriminada (Brasil, 2016). Em relação aos analgésicos e antitérmicos a preocupação é que o uso excessivo possa desenvolver uma hepatotoxicidade no indivíduo, bem como, deve-se considerar a possibilidade desses medicamentos, por possuírem efeitos paliativos, esconderem uma possível doença mais grave, visto que eles tratam sintomas (dor e febre) e não a causa da enfermidade (Vieira \& França, 2015).Além disso, o uso indiscriminado e prolongado dos anti-inflamatórios pode ocasionar malefícios como, úlcera péptica, hipertensão arterial sistêmica, insuficiência cardíaca e insuficiência renal aguda (Silva, 2017).

Não se encontrou, em trabalhos semelhantes disponíveis na literatura, dados sobre frequência de automedicação entre universitários. Apesar de ser um comportamento majoritariamente esporádico, esse dado não deve nos conduzir a uma interpretação equivocada de que se trata de uma questão irrelevante, considerando-se, por exemplo, o fato de que muitas reações adversas a medicamentos não seguem proporcionalidade direta com a dose e frequência de uso do fármaco. Ademais, esse padrão de uso de fármacos, é facilmente caracterizado como farmacoterapia paliativa, fato que nos deveria fazer refletir, em uma perspectiva de educação integral, sobre quais as dores que estão sendo "silenciadas" pelos discentes, suas origens, recorrências e a provável relação com o contexto acadêmico, o qual, com sabemos, pode ser favorecedor de sofrimento físico e emocional. Importante salientar, que esse uso indiscriminado de medicações pode ocultar doenças evolutivas, que se manifestam através da dor, resultando em um diagnóstico tardio (Gama et al., 2017).

A principal fonte de informação sobre o uso desses medicamentos foi uma associação entre internet, familiares e amigos e a bula dos medicamentos, sendo mínima a busca por um profissional de saúde capacitado. No estudo de Lopes (2014) essa prática foi justificada pelo conhecimento do produto relacionado a uso habitual, prescrição médica anterior e uso frequente na família. Aqui, vários fatores podem ser relacionados para explicar tal comportamento, muito similar à população em geral, tais como: o "imediatismo terapêutico" fortalecido pela falta de tempo na vida moderna; a dificuldade de acesso aos serviços de saúde, principalmente na rede pública; o estímulo publicitário ao consumo de medicamentos de venda livre, entre outros fatores. Entretanto, considerando as especificidades dos entrevistados, é possível supor que, por estarem em processo de formação na área, se julguem mais capazes de usar medicamentos por contra própria sem estarem sujeitos a riscos relevantes. Consideramos de fundamental importância refletir sobre estratégias de conscientização dos graduandos no que concerne aos riscos da farmacoterapia irracional.

Quando questionados acerca dos riscos à saúde por causa da utilização de medicamentos, os discentes responderam, em sua maioria, que a automedicação pode gerar riscos à saúde (80,2\%). Somente 19,8\% dos discentes alegaram não haver riscos, afirmando que, se houver conhecimento científico sobre os princípios básicos da Farmacologia, por parte do usuário, não há perigos à saúde dos indivíduos. De maneira semelhante, os alunos que consideravam a prática perigosa afirmaram, em sua maioria $(80,2 \%)$, que o desconhecimento dos princípios da Farmacologia como, por exemplo: dosagem correta, via certa, interações medicamentosas, efeitos adversos, resistência de fármacos e dependência, podem ser fatores potencialmente perigosos à saúde causando muitas vezes as tentativas de suicídio, altos níveis de toxicidades hepáticas e renais, bem como o coma e até a morte (WHO, 1998).

Esses dados reforçam o comentário anterior; ou seja, para os entrevistados, o mero conhecimento teórico de disciplinas já cursadas, e relacionadas aos medicamentos, principalmente a Farmacologia, os habilitaria a usar com segurança os medicamentos de venda livre em padrão de automedicação. Desconsidera-se toda a complexidade da farmacoterapia, na qual se entende que o sucesso terapêutico no uso de medicamentos se relaciona não somente com eficácia, mas também com a segurança 
desse uso. Ou seja, não basta que a droga seja capaz de produzir o efeito desejado, mas que não produza toxicidade relevante ao organismo do usuário. Para se chegar a esse objetivo, o uso do medicamento deve ser iniciado e acompanhado por um profissional que seja capaz de, usando conhecimento de várias disciplinas relacionadas tanto ao fármaco quanto ao organismo do usuário, interpretar os sinais e sintomas observados no paciente ao longo do tratamento de modo a tomar decisões sobre o seguimento farmacoterapêutico (WHO, 1998).

\section{Conclusão}

É possível concluir que entre os discentes a concepção do uso de fitoterápicos é errônea. Bem como, a maioria acredita que este uso não traz prejuízos para a saúde e justifica por ser um produto natural. Portanto, é evidente o despreparo dos estudantes de enfermagem acerca do tema, isto é, os discentes entrevistados possuem um perfil em termos de práticas e saberes sobre fitoterapia muito parecido com a população em geral. E provavelmente será refletido na não utilização dessa abordagem terapêutica durante a vida profissional, como é preconizado no SUS, o que ressalta a importância de desenvolverem-se estratégias de abordagem da fitoterapia ao longo da formação de profissionais de nível superior em saúde.

Em relação a automedicação, os grupos farmacológicos mais utilizados são os analgésicos e anti-inflamatórios; porém, é preocupante a utilização indiscriminada de corticóides, antibióticos e anti-hipertensivos. Sobre os riscos desta prática para a saúde, a maioria acredita trazer malefícios, e uma minoria, principalmente dos últimos períodos, relata que ao possuir conhecimento científico sobre os princípios básicos da Farmacologia e sobre a atuação do medicamento no organismo, o usuário estaria apto para uma automedicação consciente.

É necessário a realização de novas pesquisas que tenham como objetivo avaliar o conhecimento dos acadêmicos da saúde em universidades/faculdades que apresentem a fitoterapia na grade curricular. Avaliando o interesse e a disseminação de informações com comprovação científica, unidas ao conhecimento tradicional têm impacto para a sociedade. Como também, avaliar a importância de profissionais da saúde com conhecimento em fitoterapia e PICS no serviço público/atenção básica. Constata-se que é urgente a elaboração de estratégias para discussão do tema da automedicação, inclusive com produtos fitoterápicos, entre o segmento populacional avaliado, como forma de promoção da saúde e qualidade de vida de jovens universitários, mediante uma educação para além dos conteúdos curriculares obrigatórios, mas, também e sobretudo, para que os mesmos estão mais habilitados a, no futuro, quando do exercício profissional, promover a inserção da fitoterapia nos serviços de saúde e desestimular o uso irracional de medicamentos.

\section{Agradecimentos}

Agradecemos ao Professor Ednário Barbosa de Mendonça Docente do Departamento de Estatística da Universidade Estadual da Paraíba (UEPB) pela colaboração na análise estatística dos dados da pesquisa.

\section{Referências}

Badke, M, R., Heisler, E, V., Ceolin, S., Andrade, A., Budó, M, L, D., \& Heck, M, R (2017). O conhecimento de discentes de enfermagem sobre uso de plantas medicinais como terapia complementar. Revista de Pesquisa: Cuidado é Fundamental Online, [S.1.], v. 9, n. 2, p. 459-465, apr. 2017. ISSN-2175-5361. http://www.seer.unirio.br/index.php/cuidadofundamental/article/view/5442.

Barreto, B.B (2015). Fitoterapia no conteúdo dos cursos de graduação da área de saúde: importância para a formação de profissional qualificado. OnlineRepositório UNB Brasília, 2015, 150p. http://repositorio.unb.br/bitstream/10482/18613/1/2015_BenilsonBelotiBarreto.pdf.

Brasil. Agência Nacional de Vigilância Sanitária. Medicamentos fitoterápicos, 2015.

Brito, F, M., Oliveira, A, F, P., Costa, I, C, P., Andrade, C, G., Santos, K, F, O. \& Anízio, B, K, F (2017). Fitoterapia na atenção básica: estudo com profissionais enfermeiros. Revista de Pesquisa: Cuidado é Fundamental Online, [S.1.], v. 9, n. 2, p. 480-487, apr. $2017 . \quad$ ISSN 2175-5361. http://www.seer.unirio.br/index.php/cuidadofundamental/article/view/5449. doi:http://dx.doi.org/10.9789/2175-5361.2017.v9i2.480-487. 
Correa, N., Soares, M. C. F. \& Muccillo-Baisch, A. L (2018). Conhecimento do tema plantas medicinais e fitoterápicos como instrumento tecnológico na formação dos acadêmicos de enfermagem. Vittalle - Revista de Ciências da Saúde, v. $30, \quad \mathrm{n} . \quad 2 \quad$ (2018) $38-46$. https://periodicos.furg.br/vittalle/article/view/7496/5400.

Cansanção, I.F. (2018). levantamento etnobotânico de plantas medicinais utilizadas por moradores do entorno do parque nacional serra da capivara - pi. BioFarm. ISSN 1983-4209, v. 13, n. 04 (2017). http://revista.uepb.edu.br/index.php/biofarm/article/view/2924/2369.

Duarte, A., Masiero, A., Boff, P., \& Pucci, M. (2020). saberes e práticas populares no uso de plantas medicinais em espaço urbano no sul do brasil. Revista Brasileira De Agroecologia, 15(1), 13. doi:10.33240/rba.v15i1.22978

Gama, A.S., Oliveira, M.R., Beazussi, K.M., \& Gama, A.S. (2017). automedicação entre acadêmicos de enfermagem em uma instituição particular de ensino. linkscienceplace - Interdisciplinary Scientific Journal, 3, 74-85. DOI:10.17115/2358-8411/v3n2a6

Gonçalves, R. N.(2017) Práticas integrativas na atenção primária à saúde, sob a ótica da fitoterapia. Curitiba, 2017. 192 f.

instrução normativa - in no - 11, de 29 de setembro de $2016 . \quad$ Brasília, DF, set, 2016. http://pesquisa.in.gov.br/imprensa/jsp/visualiza/index.jsp?jornal=1\&pagina=99\&data=30/09/2016

MAIA, A. C. P., Paiva, P, C, B. \& Ferreira, E, C., \& Pereira, R, F, P, L (2016). A fitoterapia sob a ótica dos profissionais de saúde no Brasil nos últimos 10 anos. Gaia Scientia, v. 10, n. 4, 2016.

Maia, M. V. P (2013). O conhecimento dos acadêmicos de enfermagem a respeito do uso de fitoterápicos. UniCEUB. Online. Brasília, DF, 2013. http://repositorio.uniceub.br/bitstream/235/4500/1/2.pdf.

Mattos, G., Camargo, A., Sousa, C., \& Zeni, A.L (2018). Plantas medicinais e fitoterápicos na Atenção Primária em Saúde: percepção dos profissionais. Ciencia \& Saude Coletiva, 23, 3735-3744. https://doi.org/10.1590/1413-812320182311.23572016

MONOGRAFIA DA ESPÉCIE Matricaria chamomilla L. (= Chamomilla recutita (L.) Rauschert, CAMOMILA). Ministério da Saúde e Anvisa, Brasília, 2015. http://portalarquivos2.saude.gov.br/images/pdf/2017/setembro/11/Monografia-Camomila.pdf.

Pereira A. S., Shitsuka D.M., Parreira F.J., \& Shitsuka R (2018). Metodologia da pesquisa científica. [free e-book]. Santa Maria/RS. Ed. UAB/NTE/UFSM

Política e Programa Nacional de Plantas Medicinais e Fitoterápicos / Ministério da Saúde, Secretaria de Ciência, Tecnologia e Insumos Estratégicos, Departamento de Assistência Farmacêutica. - Brasília: Ministério da Saúde, 2016.Sampaio, L. A., Oliveira, D, R., Kerntopf, M, R., Brito Jr, F, E. \& Menezes, I, R, A. Percepção dos enfermeiros da estratégia saúde da família sobre o uso da fitoterapia. Rev Min Enferm. 2013 jan/mar; 17(1): 76-84.

Relação Nacional de Medicamentos Essenciais: Rename 2020 [recurso eletrônico] / Ministério da Saúde, Secretaria de Ciência, Tecnologia, Inovação e Insumos Estratégicos em Saúde, Departamento de Assistência Farmacêutica e Insumos Estratégicos. - Brasília: Ministério da Saúde, 2020.

Santos, L. M (2014). Ecologia de saberes: a experiência do diálogo entre conhecimento científico e conhecimento tradicional na comunidade quilombola da Rocinha. Tempus, actas de saúde colet, Brasília, V.8, N.2, P. 243-256, jun, 2014.

Silva, E. C. F., Moreira, F, R., Fortunato, L, F., Lobo, M, L, S., Quintiliano, M, G, P., Silva, T, R. \& Resende, F, A (2017). O Consumismo e a Falta de Informação Sobre Riscos do Uso de Corticoides Dispensados em uma Farmácia Privada no Município de Sete Lagoas e Matozinhos- Mg. Revista Brasileira de Ciências da Vida, [S.1.], v. 4, n. 1, jul. 2017. ISSN 2525-359X. http://jornal.faculdadecienciasdavida.com.br/index.php/RBCV/article/view/494.

Silva, F. M., Goulart, F. C., \& Lazarini, C. A (2014). Caracterização da prática de automedicação e fatores associados entre universitários do curso de Enfermagem. Revista Eletrônica de Enfermagem, Goiânia, v. 16, n. $\quad 3$, p. $\quad 644-51, \quad$ set. $2014 . \quad$ ISSN $1518-1944$. https://www.revistas.ufg.br/fen/article/view/20850/17538. doi:https://doi.org/10.5216/ree.v16i3.20850.

Vieira, A. L. \& França, G. G (2015). AS CONSEQUÊNCIAS NO CONSUMO INDISCRIMINADO DO PARACETAMOL E ORIENTAÇÃO FARMACÊUTICA À PROMOÇÃO AO USO RACIONAL. Revista Acadêmica Oswaldo Cruz, São Paulo, 2015. http://www.revista.oswaldocruz.br/Content/pdf/Edicao_06_Ariane_vieira.pdf

World Health Organization. (1998). The Role of the pharmacist in self-care and self-medication : report of the 4th WHO Consultative Group on the Role of the Pharmacist, The Hague, The Netherlands, 26-28 August 1998. World Health Organization. https://apps. who.int/iris/handle/10665/65860

Zampieron, R.G., Sheng, L.Y., Gontijo, L., \& Mueller, A (2019). Profile of the self-medication of undergraduate students in the health area from higher education institution in Sinop-MT. Scientific Electronic Archives, 12, 101-110. DOI: http://dx.doi.org/10.36560/1252019790 